\title{
Tuftelin 1 (TUFT1) Promotes the Proliferation and Migration of Renal Cell Carcinoma via PI3K/AKT Signaling Pathway
}

\author{
Hua Lin, Weifeng Zeng, Yuhang Lei, Desheng Chen and Zhen Nie* \\ Department of Urology, Xiantao First People's Hospital Affiliated to Yangtze University, Xiantao, China
}

Tuftelin 1 (TUFT1), a protein functioning distinctively in different tissues, is reported to be elevated in several types of cancers and the elevation of TUFT1 is correlated with unfavorable clinicopathologic characteristics and poor survival. However, the involvement of TUFT1 in renal cell carcinoma (RCC) remains unknown. In the current study, we investigated the role of TUFT1 in RCC and potential underlying mechanisms. RTPCR and Western blot analysis showed that both the mRNA and protein levels of TUFT1 were increased in primary RCC tissue and RCC cell lines. TUFT1 overexpression in RCC cells resulted in enhanced cell proliferation and migration while knockdown of TUFT1 by contrast decreased the growth and migration of the RCC cells, indicating TUFT1 expression is involved in RCC cell growth and migration. The involvement of TUFT1 in the epithelial-mesenchymal transition (EMT) of RCC cells was also determined by measuring the expression of EMT-related markers. Our data showed that TUFT1 overexpression promoted RCC cell EMT progression while knockdown of TUFT1 suppressed such process. Further signaling pathway inhibition assay revealed that TUFT1-induced RCC cell growth, migration and EMT was significantly suppressed by PI3K inhibitor, but not JNK or MEK inhibitors. In addition, TUFT1 overexpression enhanced

OPEN ACCESS

Edited by: Anna Sebestyén,

Semmelweis University, Hungary

*Correspondence: Zhen Nie

n_zhen@hotmail.com

Received: 12 December 2020 Accepted: 17 March 2021 Published: 19 April 2021

Citation: Lin H, Zeng W, Lei Y, Chen D and Nie Z (2021) Tuftelin 1 (TUFT1) Promotes the Proliferation and Migration of Renal Cell Carcinoma via PIJK/AKT Signaling Pathway. Pathol. Oncol. Res. 27:640936. doi: 10.3389/pore.2021.640936 the AKT phosphorylation, a key member of the PI3K signaling pathway, while PI3K inhibitor suppressed such process. Taken together, our study showed that TUFT1 expression was elevated in RCC and such elevation promoted the proliferation, migration and EMT of RCC cells in vitro, through PI3K/AKT signaling pathway. The findings of our current study imply that TUFT1 is involved in RCC tumorigenesis, and it may serve as a biomarker for RCC diagnosis and a potential target for RCC treatment.

Keywords: renal cell carcinoma, tuftelin 1, proliferation, migration, signaling pathway

\section{INTRODUCTION}

Renal cell carcinoma (RCC), with about 403,000 newly diagnosed cases and around 175,000 deaths annually, is one of the most common cancers worldwide [1]. RCC represents a heterogeneous group of cancers that are derived from renal tubular epithelial cells [2]. Although remarkable advances have been achieved in the understanding, diagnosis and treatment of this disease, the prognosis of RCC patients still remain unsatisfactory. Up to now, surgical removal still remains as the only curative approach [3-5]. For patients with advanced RCC, systemic immunomodulatory therapies using 
drugs like interferon- $\alpha$, IL-2, sunitinib and sorafenib have been possible [6-9]. Recently, the therapeutic spectrum has been broadened by several novel targeted agents, including as VEGF and mTOR inhibitors and the programmed cell death-1 (PD-1) immune checkpoint inhibitors (nivolumab, pembrolizumab and avelumab) [10-13]. Such immunomodulatory therapies, especially the targeted therapies have significantly improved objective response rate and/or median progression-free survival with reduced the adverse effects [7, 8, 14]. Despite these advances, tumor long-term responsiveness to these drugs are poor and patient survival rate is only slightly increased [15]. Therefore, the development of new drugs with better efficacy is still in need, while understanding the mechanism underlying RCC tumorigenesis would significantly benefit drug development.

Tuftelin 1 (TUFT1), a phosphorylated glycoprotein initially found in tooth enamel, plays a role in dental enamel mineralization [16]. The expression of TUFT1 is later also found in other tissues and the function of TUFT1 seems to be tissue dependent [17]. Recently, TUFT1 expression is reported to be elevated in several types of cancers including hepatocellular carcinoma (HCC), breast cancer, thyroid carcinoma and osteosarcoma [18-20]. Increased expression of TUFT1 can promote the growth and metastasis of cancer cells through distinctive pathways in different cancers [20, 21]. Moreover, TUFT1 expression is associated with unfavorable clinical outcomes and poor prognosis of HCC [20]. However, whether TUFT1 also plays a role in RCC still remains to determined.

In the current study, we demonstrated that TUFT1 expression was also increased in RCC tissue and cell lines. Further mechanistic investigation in vitro showed that TUFT1 promoted RCC cell proliferation, migration, and epithelialmesenchymal transition (EMT), through PI3K/AKT signaling pathway. The findings of our current study imply that TUFT1 is involved in RCC tumorigenesis, and it may serve as a biomarker for RCC diagnosis and a potential target for RCC treatment.

\section{MATERIALS AND METHODS}

\section{Ethical Statement and Patient Samples}

All protocols involving human specimens were reviewed and approved by the Ethics Review Board at Xiantao First People's Hospital (No. 2018H00109). Written informed consents were obtained from all participants. RCC tissues (CT) and adjacent noncancerous tissues (NT) were obtained from 10 RCC patients who underwent surgical resection at Xiantao First People's Hospital. None of the 10 patients received any anti-RCC treatment before the surgery. The clinicopathological data of the patients were listed in Supplementary Table S1. Harvested tissue samples were immediately stored at $-80^{\circ} \mathrm{C}$ till use.

\section{Cells and Plasmids}

Human normal renal proximal tubule epithelial cells (RPTEC), RCC cell lines A498 and 786-O were all purchased from ATCC, and cultured in DMEM/F12, EMEM and RPMI-1640 medium, respectively. A final concentration of 10\% FBS (Gibco, Thermo
Fisher Scientific) and antibiotics were added to all growth media. The human TUFT1 expression plasmid, designated as pTUFT1, was a kind gift from Dr. Kai $\mathrm{Hu}$ from Wuhan Institute of Virology, Chinese Academy of Sciences. TUFT1 shRNA (cat no: sc-61736-SH) and control shRNA (cat no: sc-108060) were both purchased from Santa Cruz Biotechnology.

\section{RT-PCR}

RT-PCR was performed as previously described with modifications $[18,22]$. In brief, total RNA was extracted using Trizol (Thermo Fisher Scientific) and reverse-transcribed into cDNA with FastQuant RT Kit (TIANGEN), according to the manufacturer's instructions. TUFT1 mRNA level was then semiquantified using a SYBR Green-based RT-PCR. GAPDH was used as an internal control. The primer pair used for TUFT1 detection were (forward) $5^{\prime}$-TCAGACTGTGTGGCTTTTGAG$3^{\prime}$ and (reverse) $5^{\prime}$-GTCAGCATTGTTGCTCCGAAG-3'. The primer pair used for GAPDH detection were (forward) $5^{\prime}$ GCCAAGGTCATCCATGACAACTTTGG-3' and (reverse) $5^{\prime}$ GCCTGCTTCACCACCTTCTTGATGTC- $3^{\prime}$. The relative expression of TUFT1 was calculated using the $2^{-\Delta \Delta \mathrm{Ct}}$ method.

\section{Transfection and Inhibitor Treatment}

All transfections were conducted using Lipo8000 ${ }^{\mathrm{TM}}$ Transfection Reagent (Beyotime) according to the manufacturer's instructions. In brief, plasmids were first diluted in Opti-MEM (Thermo Fisher Scientific) and then mixed with Lipo8000 ${ }^{\mathrm{TM}}$ Transfection Reagent. Afterwards, the prepared the mixture was directly added into cell culture and cultured for $48 \mathrm{~h}$. For signaling pathway inhibitor treatment, transfection mixture was removed $4 \mathrm{~h}$ post transfection and fresh medium supplemented with inhibitors were added and cells were cultured in the presence of inhibitor for another $48 \mathrm{~h}$. The following signaling pathway inhibitors were used in the current study: SP600125 (JNK inhibitor; $5 \mu \mathrm{M}$ ), LY294002 (PI3K inhibitor; $25 \mu \mathrm{M}$ ) and PD98059 (MEK inhibitor; $50 \mu \mathrm{M}$ ). All inhibitors were purchased from Selleck and used as per the manufacturer's instructions.

\section{Western Blot}

Western blot was performed as previously described with modifications [20, 23]. In brief, cells were first lysed using lysis buffer ( $150 \mathrm{mM} \mathrm{NaCl}, 1 \% \mathrm{NP}-40$ and $50 \mathrm{mM}$ Tris $\mathrm{pH} 8.0$ ) supplemented with protease inhibitor cocktail (Beyotime), and then cleared cell lysates were mixed with SDS-PAGE loading buffer (50 mM Tris- $\mathrm{HCl}, \mathrm{pH} 6.8,2 \%$ SDS, 25\% glycerol) and boiled for $10 \mathrm{~min}$. Prepared samples were then separated by a $12 \%$ SDS-PAGE gel and transferred onto a PVDF membrane. The membrane was subsequently blocked with 5\% non-fat milk for $1 \mathrm{~h}$ at room temperature. After three washes with PBST, membrane was then sequentially incubated with primary antibodies and HRP-conjugated secondary antibodies overnight at $4^{\circ} \mathrm{C}$ and $1 \mathrm{~h}$ at room temperature, respectively. Following extensive washes, membrane was developed with ECL substrate (Beyotime), according to the manufacturer's instructions. The following primary antibodies were used: rabbit anti-human TUFT1 antibody (1:1,000 dilution; Cat no: 23385-1AP; Proteintech), mouse anti-human E-cadherin antibody (1:1,000 dilution; Cat no: 60335-1-Ig; Proteintech), mouse anti-human N-cadherin antibody (1:1,000 dilution; Cat no: 66219-1-Ig; 
Proteintech), rabbit anti-human Snail (1:1,000 dilution; Cat no: 130991-AP; Proteintech), mouse anti-human AKT antibody (1:1,000 dilution; Cat no: 60203-2-Ig; Proteintech), rabbit anti-human phospho-AKT (Thr308) ( $p$-AKT (Thr308), 1:1,000 dilution; Cat no: 9275; Cell Signaling Technology), mouse anti-human phosphoAKT (Ser473) (p-AKT (Ser473), 1:1,000 dilution; Cat no: 66444-1-Ig; Proteintech) and mouse anti-human GAPDH (1:2,000 dilution; Cat no: A00227; Boster). The following secondary antibodies were used: HRP-conjugated goat anti-mouse IgG $(\mathrm{H}+\mathrm{L})$ (1:30,000 dilution; Cat no: BA1050; Boster) and HRP-conjugated goat anti-rabbit IgG $(\mathrm{H}+$ L) (1:30,000 dilution; Cat no: BA1054; Boster). Western blot band intensity was semiquantified by ImageJ (version 1.53c; National Institutes of Health, Bethesda, MD, United States) and relative protein expression was calculated using GAPDH as the reference.

\section{MTT Assay}

The cell proliferation was assessed using a MTT cell proliferation and cytotoxicity assay kit (Sangon Biotech), according to the manufacturer's instructions. In brief, cell culture medium was removed, and cells were treated with 1:1 diluted MTT solution in fresh serum-free medium for $3 \mathrm{~h}$ at $37^{\circ} \mathrm{C}$. Following incubation, MTT solvent was added into each well and incubated on an orbital shaker for $15 \mathrm{~min}$. Finally, the samples were read at OD590 $\mathrm{nm}$ with a microplate reader.

\section{Wound Healing Assay}

Cell migration was determined by wound healing assay, as previously described with modifications $[24,25]$. In brief, cells pre-seeded at $50-60 \%$ confluency were first transfected with vector, pTUFT1, control shRNA or TUFT1 shRNA, and when cells formed a monolayer, a "wound" was introduced by scratching the cell monolayer with a sterile $200 \mu \mathrm{l}$ tip. After washes with PBS to remove non-attached cells, the cell monolayer was then incubated in fresh medium and cultured for $24 \mathrm{~h}$. The cell migration was measured by the "wound healing" rate. For cells receiving inhibitor treatment, inhibitors or mock controls were introduced into the culture right after the PBS washes.

\section{Statistical Analysis}

All data were expressed as mean \pm standard deviation (SD), and all statistical analyses were performed with Prism 8 (GraphPad). Either a Mann-Whitney test or a Kruskal-Wallis test with Dunn's multiple comparisons test were used, and a $p$ value less than 0.05 was considered statistically significant.

\section{RESULTS}

\section{TUFT1 Expression Is Elevated in RCC Tissues and Cell Lines}

TUFT1 expression was previously reported to be elevated in HCC cancer tissue (CT) comparing to adjacent noncancerous tissue (NT) [20]. To check if a similar trend could be observed in RCC, we collected 10 pairs of CT and NT samples from RCC patients who underwent surgical resection and measured the TUFT1 mRNA and protein levels. To rule out possible drug interference, none of the patients had received any anti-RCC treatment before surgery. Our data showed similar results to what was reported in HCC
(Figures 1A,B,D and Supplementary Figure S1). Namely, both the mRNA and protein levels of TUFT1 was significantly increased in CT comparing to adjacent NT.

We next further investigated whether such elevation in TUFT1 expression could also be observed in RCC cell lines. TUFT1 mRNA level in one normal renal proximal tubule epithelial cells (RPTEC) and 2 RCC cell lines A498 and 786-O were measured by RT-PCR. Consistently, TUFT1 mRNA was significantly increased in both RCC cell lines (Figure 2A). Similar results were observed when the TUFT1 protein level was determined by Western blot (Figures 2B,C). Taken together, our data here indicate that TUFT1 expression is upregulated in RCC.

\section{TUFT1 Promotes RCC Cell Growth and Migration}

To investigate the impact of TUFT1 expression on RCC cell growth and migration, TUFT1 expression was either upregulated or downregulated in A498 cells, and then cell proliferation and migration were determined. As shown in Figure $\mathbf{3 A}$ and Supplementary Figure S2, transfection of pTUFT1 considerably increased TUFT1 protein expression in A498 cells. In accordance with the increased TUFT1 expression, the proliferation rate of the A498 cells with TUFT1 overexpression showed significantly enhanced cell growth (Figure 3C) and migration (Figures 3E,G), comparing to vector-transfected cells. By contrast, cells with TUFT1 shRNA transfection showed downregulated TUFT1 expression (Figure 3B). Accordingly, cells with decreased TUFT1 showed slower proliferation rate (Figure 3D) as well as slower migration (Figures 3F,H). Together, these data indicate that TUFT1 enhances RCC cell growth and migration.

\section{TUFT1 Promotes the Epithelial-Mesenchymal Transition (EMT) of RCC Cells}

EMT is a biological process allowing immobilized polarized epithelial cells transdifferentiate into motile mesenchymal cells, which exhibit enhanced invasiveness and resistance to apoptosis $[26,27]$. The activation of an EMT process is considered the critical mechanism mediating cancer metastasis [28]. The involvement of TUFT1 in the regulation of EMT in RCC cells were subsequently investigated by determining the expression of EMT-related markers (E-cadherin, N-cadherin and Snail). E-cadherin is an important adhesion molecule maintaining the epithelial phenotype while loss of E-cadherin leads to enhanced cell mobilization and activation of several EMT transcription factors [29]. Decrease of E-cadherin is considered the EMT hallmark. $\mathrm{N}$-cadherin, in contrast, is mainly expressed in non-epithelial cells and serves as an indicator of ongoing EMT [30,31]. Snail is one of the essential EMT-inducing transcription factors that facilitates a mesenchymal phenotype [32]. Our data showed that in A498 cells with TUFT1 overexpression, the expression of E-cadherin was decreased while the expression of $\mathrm{N}$-cadherin and Snail was increased, indicating that TUFT1 promoted EMT of RCC cells (Figures 4A,C). To further confirm that TUFT1 is involved in EMT of RCC cells, a downregulation of TUFT1 in A498 cells was 


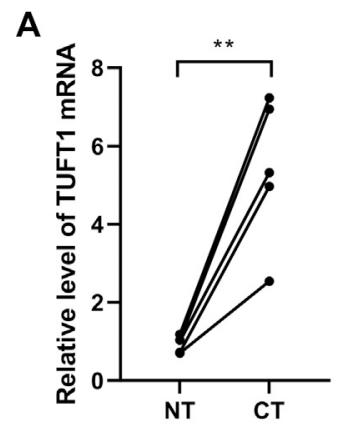

B NT CT NT CT NT CT NT CT NT CT
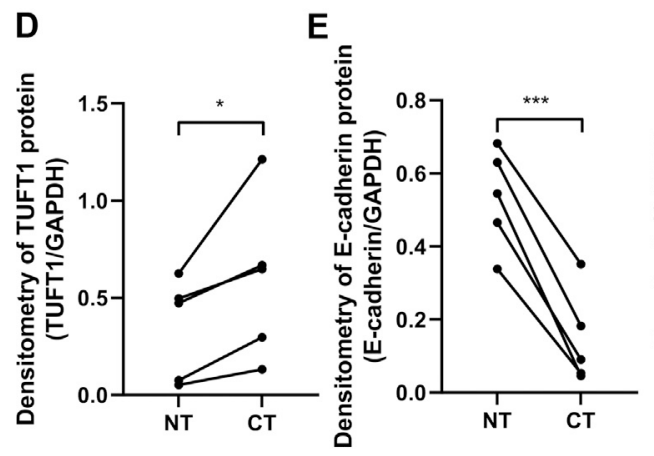

$\mathbf{F}$

NT CT NT CT NT CT NT CT NT CT

E-cadherin

GAPDH

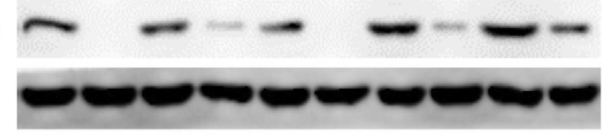

NT

G

CT
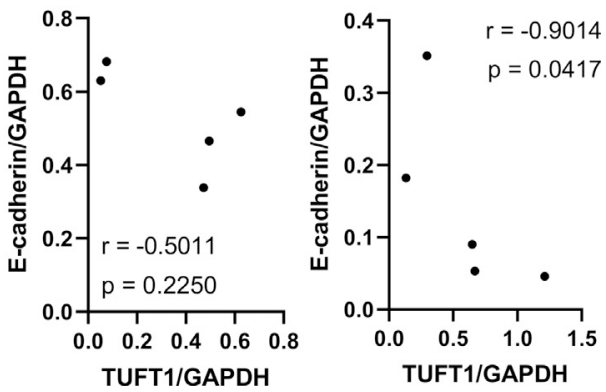

FIGURE 1 | TUFT1 expression is elevated in RCC tissue. Cancerous tissue (CT) and adjacent noncancerous tissue (NT) were collected from RCC patients and (A) the mRNA and (B, D) protein level of TUFT1 and (C, E) E-cadherin were determined by RT-PCR and Western blot, respectively $(n=5)$. (F, G) The correlation between TUFT1 and E-cadherin expression in (F) NT samples and (G) CT samples was analyzed with Spearman correlation analysis. ${ }^{\star}, p<0.05 ;{ }^{\star \star}, p<0.01 ;{ }^{\star \star \star}, p<0.001$.
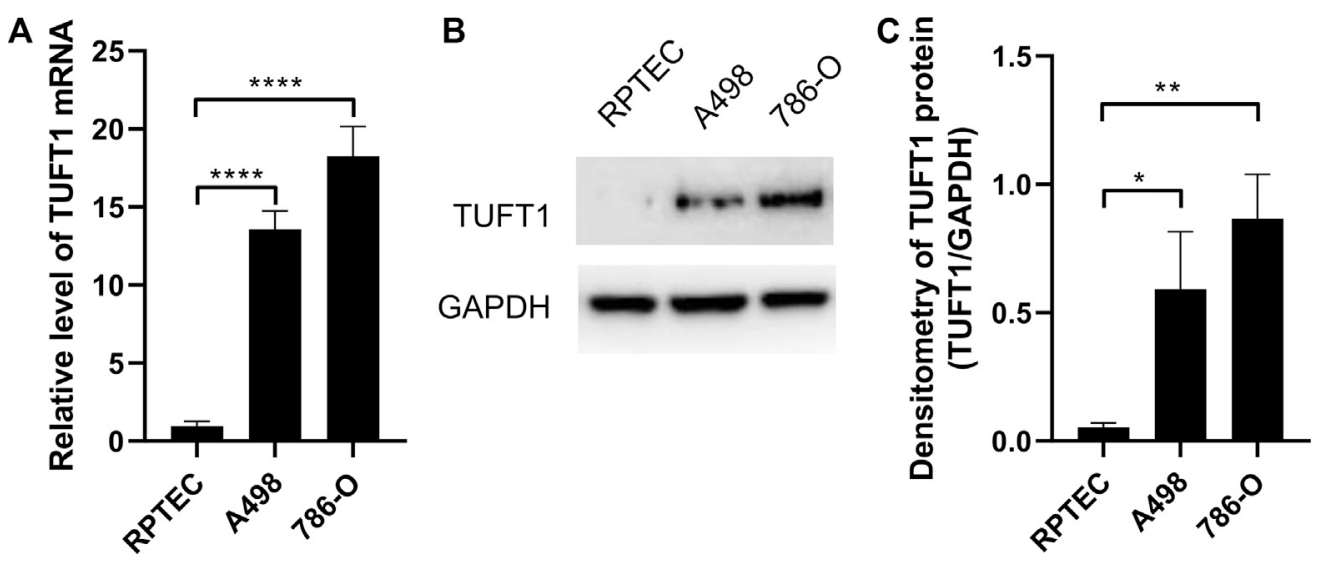

FIGURE 2 | TUFT1 expression is elevated in RCC cell lines. (A) The mRNA and (B, C) protein level of TUFT1 in normal renal epithelial cells (RPTEC) and RCC cell lines A498 and 786-O were determined by RT-PCR and Western blot, respectively. (A) Data shown are mean \pm SD of three independent experiments. (B) One representative result is shown. (C) Band intensity of $\mathbf{( B )}$ was semi-quantified by Image $\mathrm{J} .{ }^{*}, p<0.05 ;{ }^{\star *}, p<0.01 ;{ }^{* \star * \star}, p<0.0001$.

performed by TUFT1 shRNA. By contrast to TUFT1 overexpression, elevation of E-cadherin expression while decrease of $\mathrm{N}$-cadherin and Snail were observed in TUFT1 knockdown cells (Figures 4B,D). In accordance with the cell line data, E-cadherin expression was decreased in primary RCC tumor tissue than in adjacent noncancerous tissue (Figures 1C,E). Furthermore, a negative correlation between TUFT1 and E-cadherin expression was detected in the CT but not NT clinical samples (Figures 1F,G). Together, our data demonstrate that TUFT1 promotes EMT progression of RCC cells.

\section{TUFT1 Exerts Oncogenic Effects on RCC} Cells Through PI3K/AKT Signaling Pathway

We next explored possible signaling pathways underlying TUFT1-mediated proliferation, migration and EMT 


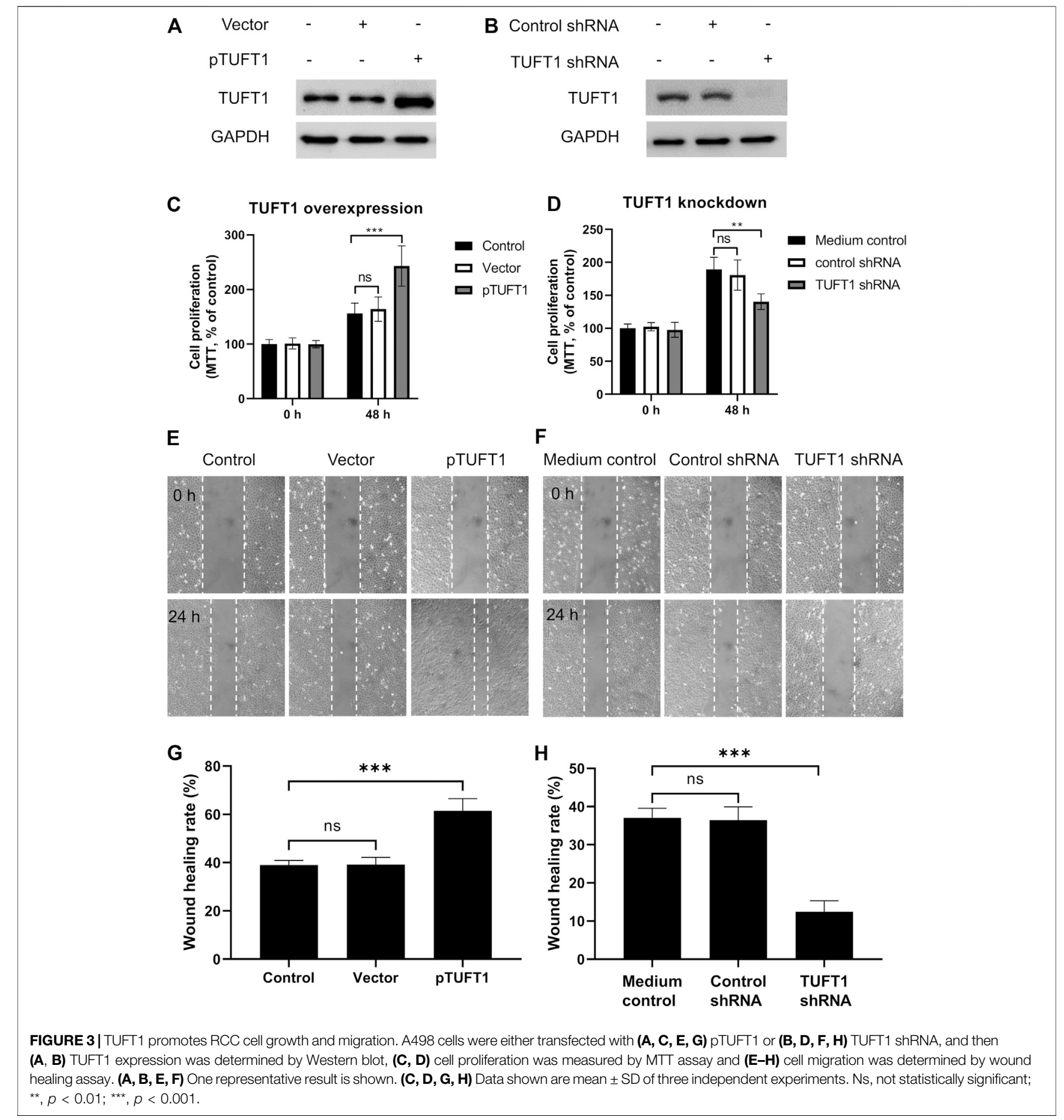

progression of RCC cells. Canonical pathways involved in cancer progression (JNK, PI3K, and AKT pathways) were investigated using signaling pathway inhibition assay [33-35]. The effects of signaling pathway inhibitors on RCC cell proliferation was first determined. As shown in Figure 5A, the overexpression of TUFT1, in consistent with our previous data, promoted cell proliferation, and the addition of inhibitor solvent, or JNK and MEK inhibitors did not induce any apparent impact on the cell growth. Of note, the enhancement of RCC cell proliferation by TUFT1 overexpression was significantly suppressed by the introduction of PI3K inhibitor into the system, indicating PI3K pathway was involved in TUFT1mediated RCC cell proliferation. Further experiments were performed to confirm whether this pathway was also involved in TUFT1-mediated migration and EMT progression of RCC cells. Similar to the proliferation assay, the migration assay 

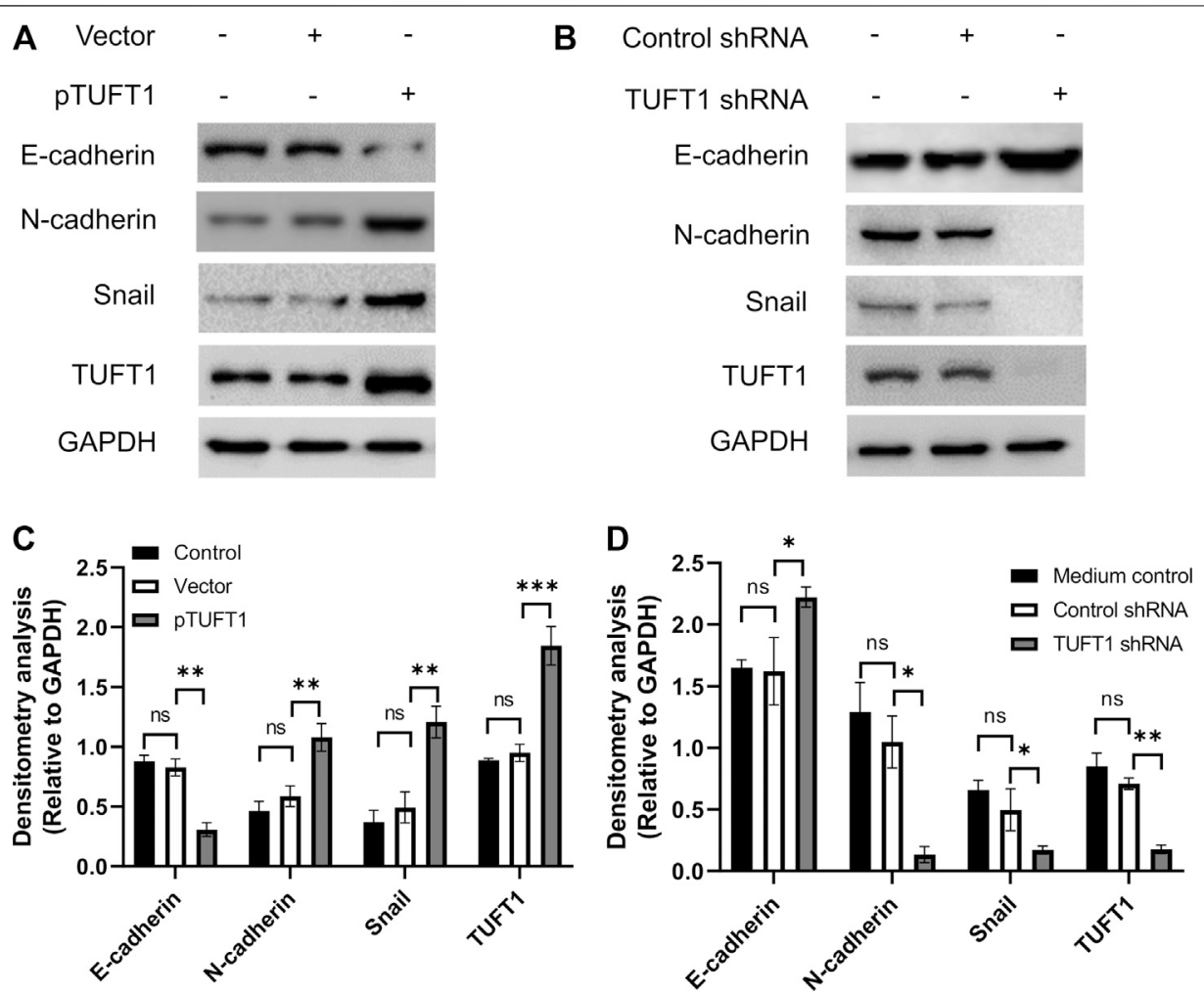

FIGURE 4 | TUFT1 promotes the EMT of RCC cells. A498 cells were first transfected with either (A) pTUFT1 or (B) TUFT1 shRNA, and then the expression of E-cadherin, N-cadherin, Snail and TUFT1 was measured by Western blot. One representative result is shown. (C, D) Band intensity analysis of (A) and (B), respectively. Data shown are mean $\pm \mathrm{SD}$ of three independent experiments. Ns, not statistically significant; ${ }^{\star}, p<0.05 ;{ }^{\star \star}, p<0.01 ;{ }^{* \star *}, p<0.001$.

showed that TUFT1 overexpression promoted RCC cell migration while the PI3K inhibitor counteracted at such enhancement (Figures 5B,C). Western blot results showed that TUFT1 overexpression did not increase the expression of AKT, a serine/threonine-specific protein kinase playing an important role in PI3K pathway but increased the phosphorylation of $\mathrm{AKT}(p-\mathrm{AKT})$ (Figure 5D). In consistent, the elevation of $p$-AKT, N-cadherin and Snail as well as the decrease of E-cadherin induced by TUFT1 overexpression were suppressed by the addition of PI3K pathway inhibitor (Figure 5D). These data here indicate that TUFT1 exerts oncogenic effects on RCC cells through PI3K/AKT signaling pathway.

Taken together, our current study has revealed that TUFT1 expression is increased in RCC tissue and cell lines both on the mRNA and protein levels. TUFT1 elevation in RCC can promote cancer cell proliferation, migration and EMT progression, via the PI3K/AKT signaling pathway.

\section{DISCUSSION}

Although remarkable advances have been made in the understanding of RCC tumorigenesis, the exact mechanism still remains elusive. TUFT1 is recently shown to be elevated in HCC and its elevation in expression promotes the progression of several types of cancers including HCC, breast cancer, thyroid carcinoma and osteosarcoma [18-20]. However, whether TUFT1 is also involved in the tumorigenesis of other tumors has not been investigated. In the current study, we have shown that TUFT1 expression was also increased in RCC. Furthermore, the increase of TUFT1 expression contributed to RCC progression by enhancing the proliferation, migration and EMT of RCC cells, through PI3K/AKT signaling pathway. The findings of our study not only advance the understanding of RCC pathogenesis, but also provide a potential diagnostic biomarker as well as treatment target.

TUFT1 is a phosphorylated glycoprotein that is initially discovered in tooth enamel and plays a role in dental enamel mineralization [16]. After synthesized in ameloblasts, TUFT1 is then secreted into the enamel matrix and accumulates at the dentin-enamel junction [36]. Later on, TUFT1 expression is detected in other non-mineralizing tissues and its functions seem to be multivariant and tissue dependent [37, 38]. It is until very recently that TUFT1 has been found to play a role in tumorigenesis [20,39]. A previous study has shown that TUFT1 is elevated in HCC and such elevation is involved in HCC growth, migration and EMT. In addition, high TUFT1 expression is correlated with unfavorable clinical outcomes and poor prognosis [20]. In consistent with the findings in HCC, we herein have demonstrated that TUFT1 expression is also increased in RCC. In addition, our study has further revealed that TUFT1 can promote the proliferation, migration and EMT of RCC cells through PI3K/AKT signaling pathway. 


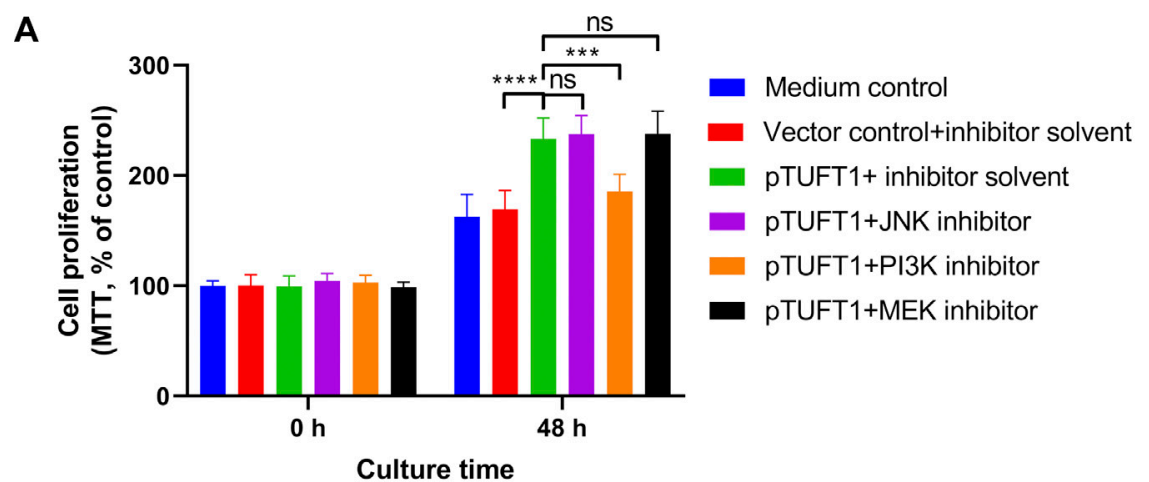

B

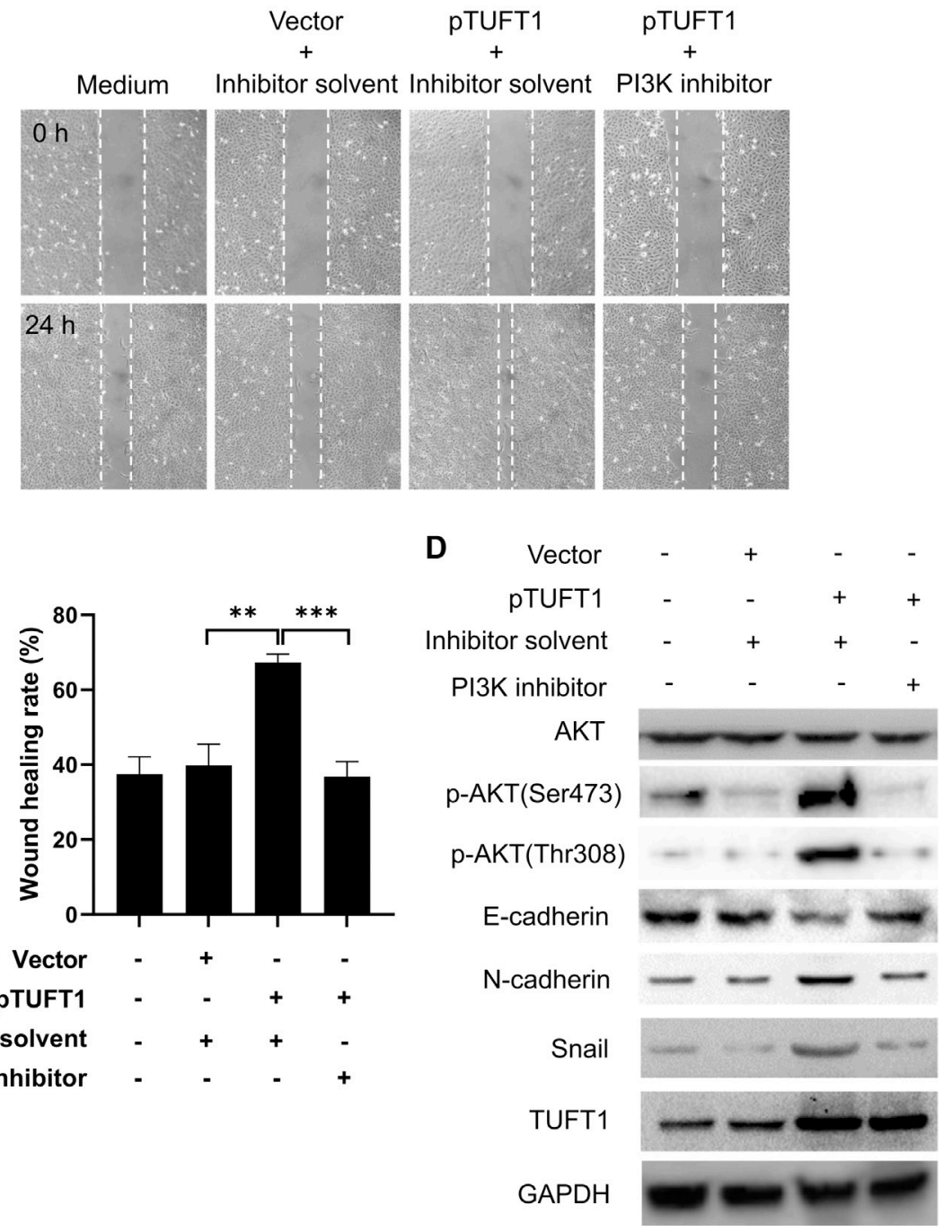

FIGURE 5 | TUFT1 exerts oncogenic effects on RCC cells through PI3K/AKT signaling pathway. RCC cells were first mock-transfected or transfected with pTUFT1 and then treated with or without specific signaling pathway inhibitors. (A) cell proliferation was measured by MTT assay. Data shown are mean \pm SD of three independent experiments. Ns, not statistically significant; ${ }^{* \star \star}, p<0.001 ;{ }^{* \star \star \star}, p<0.0001$. (B, C) Cell migration was determined by wound healing assay. One representative result is shown. (D) The expression of AKT, $p$-AKT, E-cadherin, N-cadherin, Snail and TUFT1 was determined by Western blot. One representative result is shown.

RCC is not one entity but rather a heterogenous group of different types of cancers [2]. Each type has differences in genetic characteristics, histological features and clinical phenotypes [40].
Major RCC subtypes are clear cell RCC (ccRCC, approx. 75\%), papillary RCC (approx. 15\%) and chromophobe RCC (approx. 5\%) [41, 42]. Both ccRCC and papillary RCC are believed to arise 
from the proximal tubular epithelium while chromophobe RCC and other rare subtypes are thought to arise from the distal nephron, probably from the collecting tubular epithelium [43]. In our current study, we have only checked the expression of TUFT1 in paired RCC cancer tissue and adjacent noncancerous tissue by western blot, showing that TUFT1 expression was increased in RCC tissue. However, cell specific TUFT1 expression remains unclear. In addition, we observed that TUFT1 expression was quite different between different patients. Although we do not know the reason regarding the TUFT1 expression difference, we suspect that TUFT1 expression is associated with RCC tumor stage. Of the 2 cases in our study showing lower TUFT1 expression than the rest of the cases, they are both in the early tumor stage (TNM stage $\mathrm{T} 1$ and $\mathrm{T} 2$, respectively). Similar difference in TUFT1 expression is also observed in HCC, which is reported to be associated with HCC tumor stage as well [20]. It would be interesting for further studies to investigate whether the difference in TUFT1 expression among different individuals are associated with disease progression and clinical features.

In cancer cells, many signaling pathways are abnormally activated to mediate malignant behaviors of the cells. Canonical cancer-related pathways include PI3K/AKT, JNK and MEK pathways, which increase cell proliferation and motility [44-46]. Here, we demonstrate that TUFT1 promotes AKT phosphorylation but not AKT expression in RCC cells while inhibition of PI3K pathway also suppresses the phosphorylation of AKT and RCC cell growth, migration and EMT, indicating TUFT1 exerts oncogenic functions in RCC through PI3K/AKT signaling pathway. Similarly, TUFT1 has also shown to enhance tumor cell growth and migration in HCC through the same signaling pathway [20]. However, in lung cancer cells, TUFT1 seems to enhance tumorigenesis through mTORC1 signaling pathway [39]. In addition, in breast cancer, TUFT1 promotes metastasis and chemoresistance through Rab5/Racl pathway [21]. These findings indicate that TUFT1 exerts oncogenic functions in different cancers, but the underlying mechanisms may differ. In addition, although it is beyond the scope of the current study, it would be warranted to investigate whether TUFT1 promotes tumor progression in other types of solid cancers and underlying mechanisms.

In the current study, we used signaling pathway inhibitors to determine the signaling pathway that is involved in TUFT1-enhanced RCC tumor progression. Our showed that inhibitor targeting PI3K, but not JNK or MEK pathways significantly inhibited RCC cell proliferation, migration and EMT, implying the involvement of PI3K pathway in this process. This was further confirmed by western blot that overexpression of TUFT1 could enhance the phosphorylation of Akt, a serine/threonine-specific protein kinase playing an important role in PI3K pathway. However, it must be noted that RCCs usually have high mTOR activity and inhibitors against the mTOR pathway are used to treat RCCs. Therefore, the PI3K pathway inhibitor might have affected other factors in RCC besides TUFT1. Another limitation of our current study is that all the experiments were mainly performed one cell line, A498, due to the limited research resource we could get. However, we have confirmed some of the key data using primary cancer tissue samples and another cell line 786-O.

Since TUFT1 promotes the growth, migration and EMT of RCC cells, it is possible that TUFT1 level may correlate with disease severity and/or patient survival. In fact, high TUFT1 expression is shown to correlate with poor clinical features and overall survival [20]. It is warranted to determine whether TUFT1 mRNA and/or protein level could be used as a biomarker for cancer diagnosis or stage classification. In addition, we and others have shown that inhibition of PI3K/AKT pathway could effectively suppress TUFT1-induced RCC cell proliferation and migration. It will also be interesting to investigate whether treatment strategies targeting this signaling pathway would present a novel approach for RCC treatment.

\section{DATA AVAILABILITY STATEMENT}

The original contributions presented in the study are included in the article/Supplementary Material, further inquiries can be directed to the corresponding author.

\section{ETHICS STATEMENT}

The studies involving human participants were reviewed and approved by the Ethics Review Board at Xiantao First People's Hospital. The patients/participants provided their written informed consent to participate in this study.

\section{AUTHOR CONTRIBUTIONS}

HL and ZN designed the study. HL, WZ, YL and DC performed and analyzed the data. HL and $\mathrm{ZN}$ wrote the manuscript. All authors read and approved the final manuscript.

\section{FUNDING}

The authors received no financial support for the research, authorship, and/or publication of this article.

\section{CONFLICT OF INTEREST}

The authors declare that the research was conducted in the absence of any commercial or financial relationships that could be construed as a potential conflict of interest.

\section{ACKNOWLEDGMENTS}

We thank KHE for the language editing service.

\section{SUPPLEMENTARY MATERIAL}

The Supplementary Material for this article can be found online at: https://www.por-journal.com/articles/10.3389/pore.2021.640936/ full\#supplementary-material. 


\section{REFERENCES}

1. Bray F, Ferlay J, Soerjomataram I, Siegel RL, Torre LA, and Jemal A. Global cancer statistics 2018: GLOBOCAN estimates of incidence and mortality worldwide for 36 cancers in 185 countries. CA: a Cancer J Clin (2018) 68(6):394-424. doi:10.3322/caac.21492

2. Hsieh JJ, Purdue MP, Signoretti S, Swanton C, Albiges L, Schmidinger M, et al. Renal cell carcinoma. Nat Rev Dis Primers (2017) 3(1):17009. doi:10.1038/ nrdp.2017.9

3. Van Poppel H, Da Pozzo L, Albrecht W, Matveev V, Bono A, Borkowski A, et al. A prospective, randomised EORTC intergroup phase 3 study comparing the oncologic outcome of elective nephron-sparing surgery and radical nephrectomy for low-stage renal cell carcinoma. Eur Urol (2011) 59(4): 543-52. doi:10.1016/j.eururo.2010.12.013

4. El Dib R, Touma NJ, and Kapoor A. Cryoablation vs radiofrequency ablation for the treatment of renal cell carcinoma: a meta-analysis of case series studies. BJU Int (2012) 110(4):510-6. doi:10.1111/j.1464-410x.2011.10885.x

5. Ljungberg B, Albiges L, Abu-Ghanem Y, Bensalah K, Dabestani S, FernándezPello $S$, et al. European association of urology guidelines on renal cell carcinoma: the 2019 update. Eur Urol (2019) 75(5):799-810. doi:10.1016/j. eururo.2019.02.011

6. McDermott DF, Regan MM, Clark JI, Flaherty LE, Weiss GR, Logan TF, et al. Randomized phase III trial of high-dose interleukin-2 versus subcutaneous interleukin-2 and interferon in patients with metastatic renal cell carcinoma. J Clin Oncol (2004) 23(1):133-41. doi:10.1200/ JCO.2005.03.206

7. Escudier B, Eisen T, Stadler WM, Szczylik C, Oudard S, Siebels M, et al. Sorafenib in advanced clear-cell renal-cell carcinoma. N Engl J Med (2007) 356(2):125-34. doi:10.1056/nejmoa060655

8. Motzer RJ, Hutson TE, Tomczak P, Michaelson MD, Bukowski RM, Rixe O, et al. Sunitinib versus interferon alfa in metastatic renal-cell carcinoma. $N$ Engl $J$ Med (2007) 356(2):115-24. doi:10.1056/nejmoa065044

9. Rini BI, Escudier B, Tomczak P, Kaprin A, Szczylik C, Hutson TE, et al. Comparative effectiveness of axitinib versus sorafenib in advanced renal cell carcinoma (AXIS): a randomised phase 3 trial. The Lancet (2011) 378(9807): 1931-9. doi:10.1016/s0140-6736(11)61613-9

10. Posadas EM, Limvorasak S, and Figlin RA. Targeted therapies for renal cell arcinoma. Nat Rev Nephrol (2017) 13(8):496. doi:10.1038/nrneph. 2017.82

11. Unverzagt S, Moldenhauer I, Nothacker M, Rossmeissl D, Hadjinicolaou AV, Peinemann $\mathrm{F}$, et al. Immunotherapy for metastatic renal cell carcinoma. Cochrane Database Syst Rev (2017) 5:CD011673. doi:10.1002/14651858. CD011673.pub2

12. Xu JX, Maher VE, Zhang L, Tang S, Sridhara R, Ibrahim A, et al. FDA approval summary: nivolumab in advanced renal cell carcinoma after anti-angiogenic therapy and exploratory predictive biomarker analysis. Oncol (2017) 22(3):311. doi:10.1634/theoncologist.2016-0476

13. Rini BI, Battle D, Figlin RA, George DJ, Hammers H, Hutson T, et al. The society for immunotherapy of cancer consensus statement on immunotherapy for the treatment of advanced renal cell carcinoma (RCC). J Immunotherapy Cancer (2019) 7(1):354. doi:10.1186/s40425-019-0813-8

14. Network NCC. Referenced with Permission from the NCCN Clinical Practice Guidelines in Oncology (NCCN Guidelines ${ }^{\circledR}$ ) for Non-small Cell Lung Cancer V1 (2019). , 2020. ( ) National Comprehensive Cancer Network, Inc. 2019, Plymouth Meeting, PA: 2020. ( ) National Comprehensive Cancer Network, Inc. 2019. All rights reserved (Accessed November 6).

15. Atkins MB, Clark JI, and Quinn DI. Immune checkpoint inhibitors in advanced renal cell carcinoma: experience to date and future directions. Ann Oncol (2017) 28(7):1484-94. doi:10.1093/annonc/mdx151

16. Deutsch D, Palmon A, Dafni L, Mao Z, Leytin V, Young M, et al. Tuftelin-aspects of protein and gene structure. Eur J Oral Sci (1998) 106(S1):315-23. doi:10.1111/j.1600-0722.1998.tb02192.x

17. Leiser Y, Silverstein N, Blumenfeld A, Shilo D, Haze A, Rosenfeld E, et al. The induction of tuftelin expression in PC12 cell line during hypoxia and NGFinduced differentiation. J Cell. Physiol (2011) 226(1):165-72. doi:10.1002/jcp. 22318
18. Liu W, Zhang L, Jin Z, Zhao M, Li Z, Chen G, et al. TUFT1 is expressed in breast cancer and involved in cancer cell proliferation and urvival. Oncotarget (2017) 8(43):74962-74. doi:10.18632/oncotarget.20472

19. Yu YP, He JG, Li P, Qiu NH, Wang LJ, and Feng H. TUFT1 promotes osteosarcoma cell proliferation and predicts poor prognosis in osteosarcoma atients. Open Life Sci (2018) 13(1):396-403. doi:10.1515/ biol-2018-0048

20. Dou C, Zhou Z, Xu Q, Liu Z, Zeng Y, Wang Y, et al. Hypoxia-induced TUFT1 promotes the growth and metastasis of hepatocellular carcinoma by activating the Ca2+/PI3K/AKT pathway. Oncogene (2019) 38(8):1239-55. doi:10.1038/ s41388-018-0505-8

21. Liu W, Han J, Shi S, Dai Y, and He J. TUFT1 promotes metastasis and chemoresistance in triple negative breast cancer through the TUFT1/Rab5/ Rac1 pathway. Cancer Cell Int (2019) 19(1):242. doi:10.1186/s12935-0190961-4

22. Hu K, Fu M, Wang J, Luo S, Barreto M, Singh R, et al. HSV-2 infection of human genital epithelial cells upregulates TLR9 expression through the SP1/ JNK signaling pathway. Front Immunol (2020) 11(356). doi:10.3389/fimmu. 2020.00356

23. Fu M, Hu K, Hu H, Ni F, Du T, Shattock RJ, et al. Antigenicity and immunogenicity of HIV-1 gp140 with different combinations of glycan mutation and V1/V2 region or V3 crown deletion. Vaccine (2019) 37(51): 7501-8. doi:10.1016/j.vaccine.2019.09.073

24. Liang CC, Park AY, and Guan JL. In vitro scratch assay: a convenient and inexpensive method for analysis of cell migration in vitro. Nat Protoc (2007) 2(2):329-33. doi:10.1038/nprot.2007.30

25. Pijuan J, Barceló C, Moreno DF, Maiques O, Sisó P, Marti RM, et al. In vitro cell migration, invasion, and adhesion assays: from cell imaging to data analysis. Front Cell Dev Biol (2019) 7(107). doi:10.3389/fcell.2019.00107

26. Kalluri R, and Weinberg RA. The basics of epithelial-mesenchymal transition. J Clin Invest (2009) 119(6):1420-8. doi:10.1172/JCI39104

27. Lamouille S, Xu J, and Derynck R. Molecular mechanisms of epithelialmesenchymal transition. Nat Rev Mol Cell Biol (2014) 15(3):178-96. doi:10. 1038/nrm3758

28. Thiery JP. Epithelial-mesenchymal transitions in tumour progression. Nat Rev Cancer (2002) 2(6):442-54. doi:10.1038/nrc822

29. Onder TT, Gupta PB, Mani SA, Yang J, Lander ES, and Weinberg RA. Loss of E-cadherin promotes metastasis via multiple downstream transcriptional pathways. Cancer Res (2008) 68(10):3645-54. doi:10.1158/0008-5472.Can07-2938

30. Hulit J, Suyama K, Chung S, Keren R, Agiostratidou G, Shan W, et al. $\mathrm{N}$-cadherin signaling potentiates mammary tumor metastasis via enhanced extracellular signal-regulated kinase activation. Cancer Res (2007) 67(7): 3106-16. doi:10.1158/0008-5472.Can-06-3401

31. Wang M, Ren D, Guo W, Huang S, Wang Z, Li Q, et al. N-cadherin promotes epithelial-mesenchymal transition and cancer stem cell-like traits via ErbB signaling in prostate cancer cells. Int J Oncol (2016) 48(2):595-606. doi:10. 3892/ijo.2015.3270

32. Nieto MA. The snail superfamily of zinc-finger transcription factors. Nat Rev Mol Cell Biol (2002) 3(3):155-66. doi:10.1038/nrm757

33. Vara JÁF, Casado E, de Castro J, Cejas P, Belda-Iniesta C, and González-Barón M. PI3K/Akt signalling pathway and cancer. Cancer Treat Rev (2004) 30(2): 193-204. doi:10.1016/j.ctrv.2003.07.007

34. Bubici C, and Papa S. JNK signalling in cancer: in need of new, smarter therapeutic targets. Br J Pharmacol (2014) 171(1):24-37. doi:10.1111/bph. 12432

35. Neuzillet C, Tijeras-Raballand A, de Mestier L, Cros J, Faivre S, and Raymond E. MEK in cancer and cancer therapy. Pharmacol Ther (2014) 141(2):160-71. doi:10.1016/j.pharmthera.2013.10.001

36. Delgado S, Deutsch D, and Sire JY. Evolutionary analysis of the mammalian tuftelin sequence reveals features of functional importance. Int Mol Evol (2017) 84:214-224. doi:10.1007/s00239-017-9789-5

37. Leiser Y, Blumenfeld A, Haze A, Dafni L, Taylor AL, Rosenfeld E, et al. Localization, quantification, and characterization of tuftelin in soft tissues. Anat Rec (2007) 290(5):449-54. doi:10.1002/ar.20512

38. Sliz E, Taipale M, Welling M, Skarp S, Alaraudanjoki V, Ignatius J, et al. TUFT1, a novel candidate gene for metatarsophalangeal osteoarthritis, plays a 
role in chondrogenesis on a calcium-related pathway. PLoS one (2017) 12(4): e0175474. doi:10.1371/journal.pone.0175474

39. Kawasaki N, Isogaya K, Dan S, Yamori T, Takano H, Yao R, et al. TUFT1 interacts with RABGAP1 and regulates mTORC1 signaling. Cell Discov (2018) 4(1):1-16. doi:10.1038/s41421-017-0001-2

40. Rini BI, Campbell SC, and Escudier B. Renal cell carcinoma. The Lancet (2009) 373(9669):1119-32. doi:10.1016/s0140-6736(09)60229-4

41. The Cancer Genome Atlas Research Network. Comprehensive molecular characterization of clear cell renal cell carcinoma. Nature (2013) 499(7456): 43. doi:10.1038/nature12222

42. Davis CF, Ricketts CJ, Wang M, Yang L, Cherniack AD, Shen H, et al. The somatic genomic landscape of chromophobe renal cell carcinoma. Cancer cell (2014) 26(3):319-30. doi:10.1016/j.ccr.2014.07.014

43. Cairns P. Renal cell carcinoma. Cbm (2011) 9(1-6):461-73. doi:10.3233/cbm2011-0176
44. Altomare DA, and Testa JR. Perturbations of the AKT signaling pathway in human cancer. Oncogene (2005) 24(50):7455-64. doi:10.1038/sj.onc.1209085

45. Roberts PJ, and Der CJ. Targeting the Raf-MEK-ERK mitogen-activated protein kinase cascade for the treatment of cancer. Oncogene (2007) 26(22): 3291-310. doi:10.1038/sj.onc.1210422

46. Sanchez-Vega F, Mina M, Armenia J, Chatila WK, Luna A, La KC, et al. Oncogenic signaling pathways in the cancer genome atlas. Cell (2018) 173(2): 321-37.e10. doi:10.1016/j.cell.2018.03.035

Copyright (c) 2021 Lin, Zeng, Lei, Chen and Nie. This is an open-access article distributed under the terms of the Creative Commons Attribution License (CC BY). The use, distribution or reproduction in other forums is permitted, provided the original author(s) and the copyright owner(s) are credited and that the original publication in this journal is cited, in accordance with accepted academic practice. No use, distribution or reproduction is permitted which does not comply with these terms. 